\title{
Rydberg noisy dressing and applications in making soliton molecules and droplet quasicrystals
}

\author{
Mohammadsadegh Khazali $\odot$ \\ Institute for Quantum Optics and Quantum Information of the Austrian Academy of Sciences, Innsbruck 6020, Austria; \\ Department of Physics, Sharif University of Technology, Tehran 14588, Iran; \\ and Department of Physics and Astronomy, Aarhus University, DK-8000 Aarhus C, Denmark
}

(Received 29 June 2020; accepted 15 July 2021; published 5 August 2021)

\begin{abstract}
The current advances in the field of ultracold atoms and atomic traps recall new controllable long-range interactions. These interactions are expected to extend the range of realizable quantum algorithms as well as provide new control mechanisms for the new types of quantum matters. This Letter presents special interatomic interactions between Rydberg-dressed atoms by manipulating the lasers' linewidth. The proposed interaction features a hybrid spatial profile containing plateaus and Gaussian peaks. Combined with dynamic control over the sign and strength of individual interaction elements, the Rydberg noisy-dressing $(\mathrm{RnD})$ scheme provides a valuable interaction toolbox for quantum technology. As an example, RnD's applications in making stable gigantic three-dimensional soliton molecules and in the formation of quasiperiodic droplet crystals are discussed.
\end{abstract}

DOI: 10.1103/PhysRevResearch.3.L032033

A strong long-range interaction of highly excited atoms leads to a wide range of applications in quantum technology and science [1-14]. However, their radiative decay has prevented the observation of long-time excitation dynamics or coherent interaction effects on atomic motion. Furthermore, the diverging spatial scaling of the Rydberg interaction $\left(\propto r^{-3}\right.$ or $r^{-6}$ ) was limiting the range of applications. Lightly dressing atoms with Rydberg states [15-21] promises enhanced lifetimes while providing sizable interactions. Rydberg dressing forms a plateau-type interaction, where the atoms within the blockade volume would experience a Kerr-type interaction [22,23]. This type of interaction has led to interesting applications in macroscopic entanglement generation and frequency metrology [21-26], many-body physics in quantum gases [27-32], synthetic quantum magnets [33-35], and in quantum computation [36-38].

By adding laser linewidths to the dynamics of the inresonance Rydberg dressing scheme [19], this Letter shows that laser noise could be used as a controlling knob for manipulating the interaction features. Remarkably, the possibility of changing the interaction sign by a laser linewidth opens different insights for interactions in atomic, molecular, and optical (AMO) physics with exclusive attractive or repulsive natures. For example, the absence of a repulsive isotropic dressing interaction in alkaline atoms [39] could now be provided by the Rydberg noisy-dressing $(\mathrm{RnD})$ interaction. The $\mathrm{RnD}$ interaction features hybrid interaction profiles containing soft cores, barriers, and wells. Having dynamical control over the sign, strength, and distance of individual elements in the profile provides a valuable toolbox for the implementation of

Published by the American Physical Society under the terms of the Creative Commons Attribution 4.0 International license. Further distribution of this work must maintain attribution to the author(s) and the published article's title, journal citation, and DOI. quantum technology. A shell-type interaction provided with $\mathrm{RnD}$ could be used for addressing the desired interacting sites in atomic lattices [20,40-47], ideal for the implementation of quantum annealing [48], quantum simulation [49], and spinice formation [33]. The $\mathrm{RnD}$ of Bose-Einstein condensates (BECs) opens research perspectives where this Letter discusses in detail its application in making droplet quasicrystals as well as making stable giant soliton molecules.

Solitons are macroscopic quantum objects maintaining their integrity under self-trapped nonlinearities, with the three-dimensional (3D) matter version being proposed by Rydberg dressing [27]. Soliton molecules are bound states of solitons $[50,51]$ that are a macroscopic version of matter molecules with analogies in different properties such as vibration, synthesis, and dissociation [52], providing a simulating laboratory for quantum chemistry. In telecommunications applications, optical soliton molecules improve the information transfer rate beyond the fundamental threshold [50,53]. Hence, making stable soliton molecules has garnered significant interest in the last decade [50-52,54-60]. The interaction of solitons is effectively the interaction of their self-generated potential wells created under a nonlinear interaction. The interference-type forces between solitons are extremely sensitive to their relative phases. This would hinder the formation of stable solitonic molecules [see Supplemental Material (SM) [61]). The RnD scheme is applied here for designing the phase-independent self-generated potential featuring an inner soft-core attraction supporting the 3D selftrapped soliton, an outer repulsive shell (barrier) preventing soliton fusion, and a second attractive layer (well) used for completing the bound state resulting in giant stable soliton molecules. In this scheme, the distance and size of the individual solitons in the molecule can be controlled dynamically with a laser adjustment.

Rydberg-dressed atoms have been proposed for making droplet crystals $[29,62]$ where individual atoms in the lattice are replaced by droplets containing many atoms. In the new 

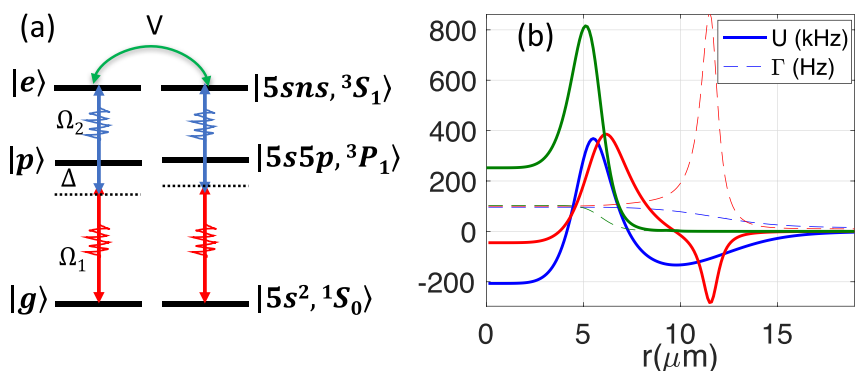

FIG. 1. Rydberg noisy-dressing (RnD) interaction. (a) Level scheme of two-photon in-resonance dressing of $\mathrm{Sr}$ atoms under an electromagnetically induced transparency (EIT) condition with noisy lasers. (b) Sample spatial profiles of the RnD interaction containing soft core, barrier, and well elements. These elements could be controlled individually by manipulating the laser parameters. The loss rate per atom $\Gamma$ is plotted by dashed lines (in $\mathrm{Hz}$ ). These interactions are used in making soliton molecules and droplet quasicrystals in this work. Laser parameters are explained in Figs. 4 and 5.

arrangement, each atom only contributes to a small fraction of the droplet's energy. Therefore, quantum tunneling of an atom between lattice sites is associated with a small energy cost. Tuning the interaction strength, droplet crystals could reveal simultaneous superfluidity and crystalline order, forming an exotic quantum matter called a supersolid [28-30,63,64]. The current Letter shows that adding Gaussian peaks to the conventional plateau structure with the $\mathrm{RnD}$ scheme, diverges the exclusive triangular structure in droplet crystals to a variety of quasiperiodic orders containing more than one tile for tessellation. These tiles in quasicrystals have $n$-fold rotational symmetry containing, e.g., $n=4,5,7,8$, 9-fold symmetries that are forbidden in classical crystalline orders [65]. Hence quasicrystals contain spatial patterns that are neither periodic as classical crystals nor totally disordered. As its classical counterpart [66-71], it is expected that the quasiordering of droplet crystals will enrich the characteristics of this quantum matter.

The level scheme of Fig. 1(a) is considered in an ultracold gas of $N$ three-level strontium atoms that undergo in-resonance two-photon excitation to the highly excited Rydberg state $|e\rangle$. The associated Hamiltonian for each atom is $H_{i}=\frac{\Omega_{1}}{2}\left(\hat{\sigma}_{g p}^{i}+\hat{\sigma}_{p g}^{i}\right)+\frac{\Omega_{2}}{2}\left(\hat{\sigma}_{e p}^{i}+\hat{\sigma}_{p e}^{i}\right)-\Delta \hat{\sigma}_{p p}$, where $\sigma_{\alpha, \beta}=$ $|\alpha\rangle\langle\beta|, \Omega_{1,2}$ are the Rabi frequencies of two transitions, and $\Delta$ is the laser detuning from the intermediate state. With negligible Rydberg decay rates, the system would follow the dark eigenstate $|d\rangle \propto \Omega_{2}|g\rangle-\Omega_{1}|e\rangle$ with zero light shift. In the limit of $\frac{\Omega_{1}}{\Omega_{2}} \ll 1$ ground-state atoms will be partially dressed with Rydberg states with a population of $P_{e}=\left(\frac{\Omega_{1}}{\Omega_{2}}\right)^{2}$. The effective two-body interaction $V_{i j}=C_{6} / r_{i j}^{6} \sigma_{e e}^{i} \sigma_{e e}^{j}$ is a van der Waals interaction between Rydberg atoms that is a function of interatomic distance $r_{i j}$. The interaction of $n^{3} S_{1}$ Rydberg atoms is repulsive with a van der Waals coefficient being in the range of $18 \mathrm{kHz} \mu \mathrm{m}^{6}<C_{6} / 2 \pi<11.6 \mathrm{THz} \mu \mathrm{m}^{6}$ for principal numbers $24<n<100$. This strong interaction could exceed atom-light coupling over several micrometers of interatomic separations. Furthermore, different decoherence terms including spontaneous emission from Rydberg $\sqrt{\gamma_{r}}|p\rangle\langle e|$ and intermediate level $\sqrt{\gamma_{p}}|g\rangle\langle p|$ are encountered,
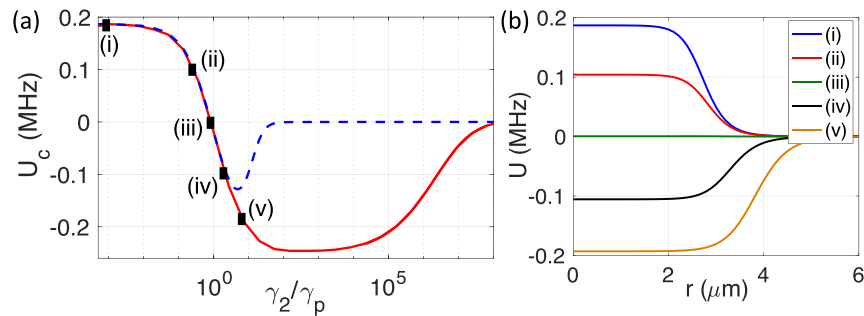

FIG. 2. Switching between an attractive and repulsive interaction with the noise knob. (a) represents the evolution of soft-core interaction strength $U_{c}$ for the case with (solid line) and without (dashed line) laser locking as a function of laser noise $\gamma_{2}=\gamma_{1}$. Here, $\Omega_{1}$ is tuned to fix the loss rate per atom to $\Gamma=100 \mathrm{~Hz}$. (b) Spatial profiles of the $\mathrm{RnD}$ interaction are plotted for the marked laser dephasing rates in (a). Laser parameters are $\Delta / 2 \pi=10 \mathrm{MHz}, \Omega_{2} / 2 \Delta=1$, targeting $n=100$ of Sr atoms.

where $\gamma_{p} / 2 \pi=7.6 \mathrm{kHz}$ in strontium and $\gamma_{r}$ can be found in Ref. [72]. The effects of the lasers' linewidth are considered with dephasing rates $\gamma_{1}$ and $\gamma_{2}$ corresponding to 689-nm $\left(\Omega_{1}\right)$ and 319-nm $\left(\Omega_{2}\right)$ lasers, respectively. The effects of laser locking included by $\gamma_{\text {Lock }}$, ranging between $\left|\gamma_{1}-\gamma_{2}\right|$ and $\left|\gamma_{1}+\gamma_{2}\right|$ for the cases of locking out of phase up to the worst case of in-phase fluctuations, see SM. Considering the steady state of two interacting atoms $\rho_{i j}$ [61], the total interaction would be given by the sum of the binary interactions

$$
\bar{U}\left(r_{i j}\right)=\operatorname{Tr}\left[\hat{\rho}_{i j}\left(\hat{H}_{i}+\hat{H}_{j}+\hat{V}_{i j}\right)\right] .
$$

In the following, the background interaction-independent light shift $\bar{U}(r=\infty)$ that only generates a constant phase, is subtracted to present the effective interaction $U(r)=\bar{U}(r)-$ $\bar{U}(\infty)$. The standard Rydberg-dressing interaction features a plateau shape with the soft-core radius $R_{c}$. The softcore radius is the distance at which the interaction-induced laser detuning equals the effective laser frequency bandwidth $P_{e}^{2} V_{\left(R_{c}\right)}^{2}=\left(\frac{\Omega_{1} \Omega_{2}}{2 \Delta}\right)^{2}+\gamma_{1}^{2}+\gamma_{2}^{2}$. The strong interaction within the soft core makes the atoms out of resonance with the laser. Hence the atoms experience a collective light shift generating a Kerr-type interaction [22,73].

There are different decoherence sources in this system. The Rydberg interaction disturbs an individual atom's dark state, populating a short-lived intermediate state $|p\rangle$, and hence increases the loss rate per atom $\Gamma=\operatorname{Tr}\left[\hat{\rho}_{i}\left(\gamma_{p} \hat{\sigma}_{p p}+\gamma_{r} \hat{\sigma}_{e e}\right)\right]$ within the soft core [see Fig. 1(b)]. Strong, unlocked laser dephasing can also disturb the dark state even for noninteracting atoms. However, locking the lasers protects the dark-state form dephasing rates smaller than the EIT bandwidth [see Fig. S1(c) in SM]. While laser noise is treated as a destructive effect, here the linewidths of locked lasers are used as a controlling parameter to manipulate the strength and design the spatial shape of the interaction. Figure 2 shows that for a constant loss rate per atom $\Gamma=100 \mathrm{~Hz}$, increasing the laser noise $\gamma_{2}$ could result in a sign change that happens at $\gamma_{2}=\gamma_{p}$ [see Fig. 2(a) and SM].

Enriching the interaction profile with extra peaks extends the applications with examples provided below. Applying adequate laser dephasing generates a first peak at the inner-peak distance given by $V^{2}\left(R_{i P}\right)=\Delta^{2}+\frac{\gamma_{2}+\gamma_{p}+\gamma_{r}}{4 \gamma_{r}} \Omega_{2}^{2}+\frac{\left(\gamma_{2}+\gamma_{p}+\gamma_{r}\right)^{2}}{4}$ 

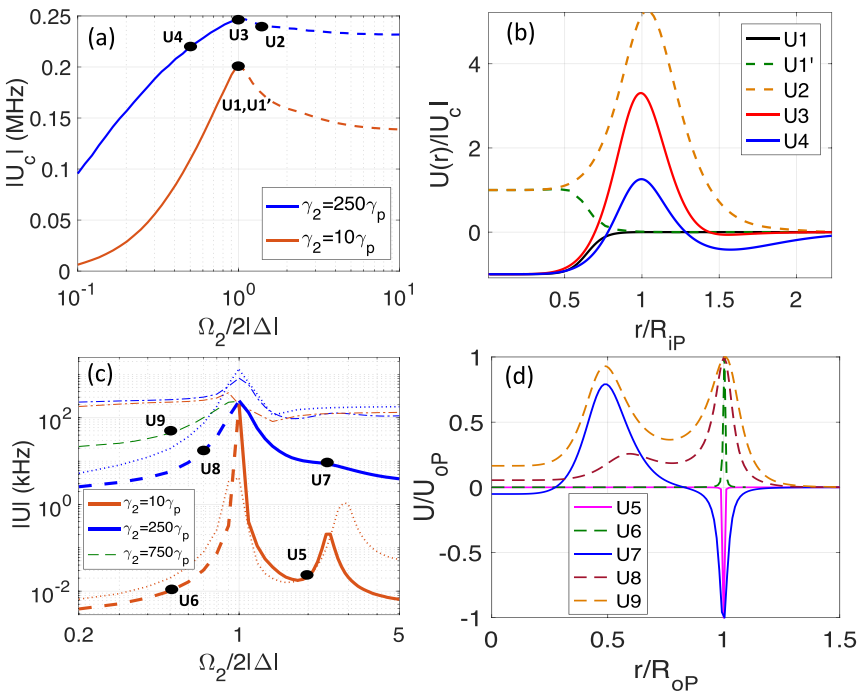

FIG. 3. Controlling the elements of interaction with the noise knob. (a), (c) The scalings of the interactions' magnitude of the soft core $\left|U_{c}\right|$ are presented by thick solid (dashed) lines associated with positive (negative) detuning. The maximum loss rate over the profile is fixed to $\Gamma=100 \mathrm{~Hz}$. (c) The interaction magnitude of the (inner) outer peak $\left(\left|U_{i P}\right|\right)\left|U_{o P}\right|$ is shown by (dotted) dotted-dashed lines. The interaction profiles for the marked points are presented in (b) and (d). Here, $\Delta / 2 \pi=10 \mathrm{MHz}, 0.1<\Omega_{1} / 2 \pi<2 \mathrm{MHz}, n=100, \gamma_{1}=\gamma_{2}$, and lasers are locked.

[see Fig. 3(b)]. The effective line broadening leads to blockade leakage. This would result in the increase of the two-atom Rydberg excitation $\rho_{e e, e e}$ and lead to a direct Rydberg interaction $\frac{C_{6}}{r^{6}} \rho_{e e, e e}$ [see Fig. S3(b)]. Note that blockade leakage only increases $\rho_{e e, e e}$, leaving Rydberg and the intermediate-state populations $\rho_{e e}, \rho_{p p}$ unaltered at the regime of interest. Therefore, the loss does not increase over this peak. By reducing $\Omega_{2} / 2 \Delta$ or increasing $\gamma_{2}$, the soft core could extend beyond the inner peak, making profiles such as $U_{4}$ in Fig. 3(b). In this case, the peak effectively splits the soft core, making an inner soft-core radius of $R_{i C}=R_{c}$ and the outer soft-core radius given by $V\left(R_{o C}\right)=2 \delta_{\mathrm{EIT}}$, where $\delta_{\mathrm{EIT}}=\frac{\Omega_{2}^{2}}{4|\Delta|}$ is the EIT bandwidth $[9,19,74]$.

An outer peak can be formed via a two-body interactioninduced resonance. The limited increase of the intermediate and Rydberg levels' population at the outer-peak position enhances the loss rate (see SM). This peak occurs in two regimes of parameters $\left(\Omega_{2}>2|\Delta|\right.$ with $\left.\Delta>0\right)$ and $\left(\Omega_{2}<2|\Delta|\right.$ with $\Delta<0)$ [see Fig. 3(c)]. The position of the outer peak would be at $V\left(R_{O P}\right)=\frac{2 \Delta \Omega_{2}^{2}}{\left(\Omega_{2}^{2}+\gamma_{p}^{2}-4 \Delta^{2}\right)}($ see $\mathrm{SM})$.

The main features of the $\mathrm{RnD}$ interaction profile are discussed in Fig. 3, for the case where the maximum loss over the profile is fixed to $\Gamma=100 \mathrm{~Hz}$. The soft cores and outer peak are formed by the interaction-induced collective light shift. Therefore, the interaction sign in these elements is defined by the sign of $\Delta$ and the value of $\gamma_{2}$. Being interested in the parameter regime $\gamma_{2} \gg \gamma_{p}$, the positive (negative) interaction is generated by $\Delta<0(\Delta>0)$ shown by the dashed (solid) lines in Fig. 3. The inner peak is directly generated by the van der Waals interaction and hence its sign follows the sign of $C_{6}$. In Fig. 3 , there is an optimum interaction-to-loss ratio $U_{c} / \Gamma=2000$ at $\Omega_{2} / 2 \Delta=1$.

While the magnitudes of $\left|U_{c}\right|$ and $\left|U_{i P}\right|$ strongly depend on $\gamma_{2}$ and $\Omega_{2}$, the outer-peak strength $\left|U_{o P}\right|$ mainly depends on $\Delta$ at the regime of interest. This feature could be used to isolate the outer peak [see $U_{5}, U_{6}$ in Fig. 3(d)], forming a shelltype interaction profile in 3D with applications in controlled interactions among the desired sites in optical lattices.

First application: Stable soliton molecules could ideally be formed by the designed red/blue RnD interactions shown in Fig. 1(b). The inner soft core forms 3D stable solitons. The positive inner peak (barrier) preserves the integrity of the molecule's elements over the synthesis process, or in the case of strong vibration. To avoid the conventional phasedependent interaction, an outer shell in the form of the potential well is developed outside the barrier for an intersolitonic attraction. The stability of the molecules formed by the red/blue potentials of Fig. 1(b) is discussed in Fig. 4. Figures 4(a) and 4(b) represent the total energy of a single and molecule solitons as a function of an individual soliton's Gaussian half width at half maximum (HWHM) and intersolitonic distance, respectively. The dressing energy $E_{\text {dress }}=$ $\sum_{i<j} U\left(r_{i j}\right)$ is numerically calculated using the Marsaglia polar method for Gaussian random sampling averaging over 100 trials. Adjusting the loss and interaction strength by manipulating $\Omega_{1}$ does not alter the interaction's spatial profile. The scaling of the soft-core (well) interaction $U_{\mathrm{c}}\left(U_{\text {well }}\right)$ as a function of the maximum loss rate over the profile $\Gamma$ and the corresponding value of $\Omega_{1}$ are plotted in Fig. 4(c). The range of allowed atom numbers in the molecule shown in Fig. 4(d) is determined by stability and observability conditions. The minimum atom number $N_{\min }$ is defined by the condition that the energy of attractive forces $\left(E_{\text {dress }} \propto N^{2}\right)$ must overcome the energy of repulsive forces $\left(E_{k} \propto N\right)$. The maximum atom number $N_{\max }$ is defined by the condition of not losing any atom with $37 \%$ Poisson probability over two molecule oscillation cycles' period $t_{2}$ (i.e., $N_{\max } \Gamma t_{2}=1$ ). The molecule oscillation frequency is defined by $\omega_{\mathrm{mol}}=\sqrt{\frac{\hbar U_{\mathrm{mol}}}{N m \sigma_{\mathrm{osc}}^{2}}}$, where $U_{\text {mol }}$ and $\sigma_{\text {osc }}$ are the depth and the HWHM of the molecule potential well and $N \times m$ is the molecule mass. The main advantage of the blue potential that results in a heavier molecule comes from the controlled loss rate over the attractive well.

The molecule synthesis formed by the blue potential is simulated in Fig. 4(e). In the zero-temperature limit the dynamics are governed by the Gross-Pitaevskii equation (GPE) $i \hbar \partial_{t} \psi(x)=\left[-\frac{\hbar^{2} \nabla^{2}}{2 m}+g|\psi|^{2}+\hbar W(x)\right] \psi(x)$, where $W(x)=$ $\int U\left(x-x^{\prime}\right)\left|\psi\left(x^{\prime}\right)\right|^{2} d x^{\prime}$ is the mean-field interaction caused by the nonlocal long-range $\mathrm{RnD}$ interaction $\hbar U, m$ is the atomic mass, and $g=\frac{4 \pi \hbar^{2} a}{m}$ is the local interaction caused by the $s$-wave scattering of atoms with a scattering length of $a=$ $96 a_{0}=5088 \mathrm{pm}$ [75]. The initial states of the left $(l)$ and right $(r)$ solitons are given by $\psi_{0}(x)=A_{r} \operatorname{sech}\left[\sigma_{r}\left(x-x_{0}\right)\right] e^{i v_{r} x}+$ $A_{l} \operatorname{sech}\left[\sigma_{l}\left(x+x_{0}\right)\right] e^{i v_{l} x}$ that is normalized to particle numbers $\int\left|\psi_{0}\right|^{2} d x=N$.

Second application: Quasiordered droplet crystals could be formed by the green RnD potential depicted in Fig. 1(b). Adding a Gaussian peak to the conventional plateau profile diverges the droplet crystal's pattern that was exclusively triangular. This could be seen by looking at the time 

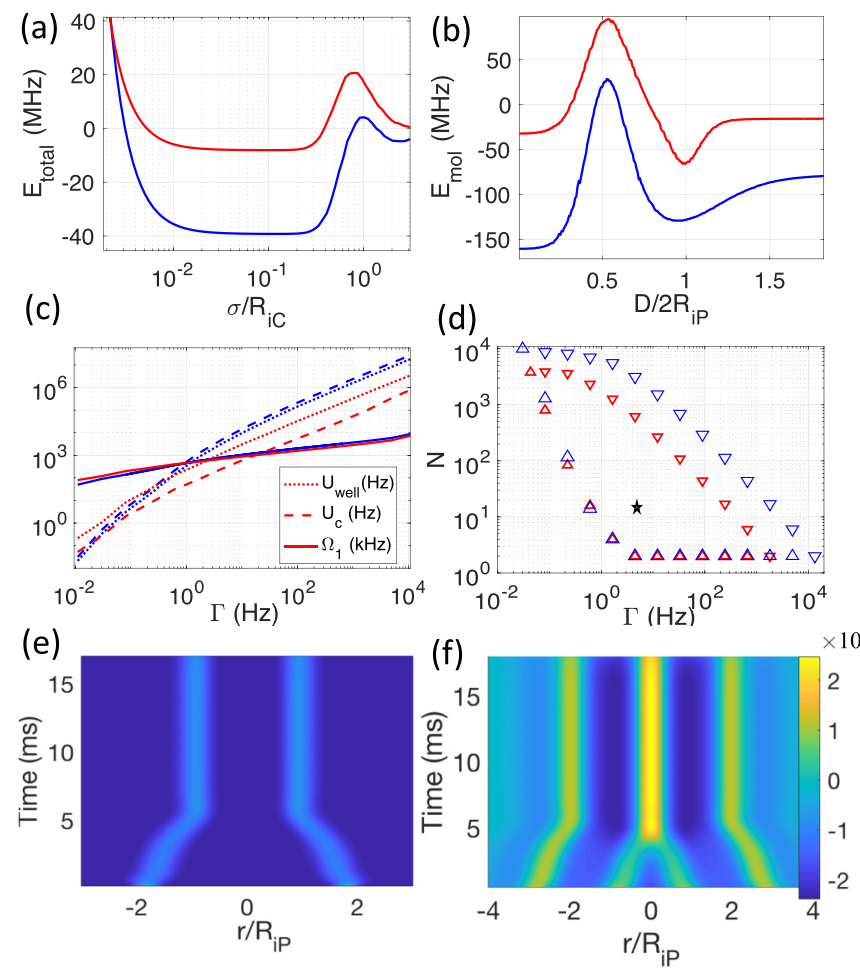

FIG. 4. Stability of 3D soliton molecules formed by the red/blue potentials of Fig. 1(b), distinguished by the same color coding. The total energy of a single soliton (a bisoliton molecule) with 20 (40) atoms is plotted as a function of the HWHM of soliton's Gaussian profile $\sigma$ (intersolitonic distance $D$ ) in (a) [(b)]. The same interaction profiles of Fig. 1(b) would scale in magnitude by manipulating $\Omega_{1}$. The scaling of the soft-core (well) interaction $U_{c}$ $\left(U_{\text {well }}\right)$ as a function of the maximum loss rate over the profile $\Gamma$ and the corresponding $\Omega_{1}$ are plotted in (c) with $2.5<R_{i C}<5 \mu \mathrm{m}$. (d) The minimum (maximum) atom numbers required for stable molecule formation are plotted by upward (downward) triangles as a function of $\Gamma$ (see the text for more details). The molecule synthesis, with parameters shown by the star sign in (d), is presented as the time evolution of (e) $|\psi|^{2}$ and (f) the mean-field interaction $W$. Laser parameters in red (blue) curves of Fig. 1(b) are $\gamma_{1}=$ $\gamma_{2}=100(1000) \gamma_{p}, \Omega_{1} / 2 \pi=450(330) \mathrm{kHz}, \Omega_{2} / 2 \Delta=2.5(0.3)$, $\Delta / 2 \pi=2(10) \mathrm{MHz}, n=100$, and the lasers are locked out of phase. The solitons' HWHM in (b)-(d) are $\sigma=0.2 R_{i C}$.

evolution of the quasiparticle excitations $\delta \psi_{\mathbf{k}}(\mathbf{r})=$ $u_{k} e^{i \mathbf{k} \cdot \mathbf{r}-i E_{b}(k) t}+\bar{v}_{k} e^{-i \mathbf{k} \cdot \mathbf{r}+i E_{b}(k) t}$ that is determined by the Bogoliubov spectrum $\omega_{b}^{2}(k)=\frac{\hbar^{2} k^{2}}{2 m}\left(\frac{\hbar^{2} k^{2}}{2 m}+2 \rho g+2 \rho \hbar \tilde{U}(k)\right)$, where $\tilde{U}(k)$ is the Fourier transform of the dressing interaction and $\rho$ is the density of atoms. The strong soft-core interaction in Rydberg-dressed atoms results in a roton unstable point that is a local minimum of the spectrum with an imaginary energy $\omega_{b}\left(k_{\text {roton }}\right)=i \beta$. Therefore, the excitation around the roton instability $k_{\text {roton }}$ would grow exponentially with the rate $\beta$. This would form a spatial periodic structure with a lattice constant of $a=\frac{2 \pi}{k_{\text {roton }}}$. The soft core in conventional Rydberg dressing forms a single roton unstable point and hence generates a triangular lattice [29]. In this work, adding a Gaussian to the soft core with the RnD technique generates
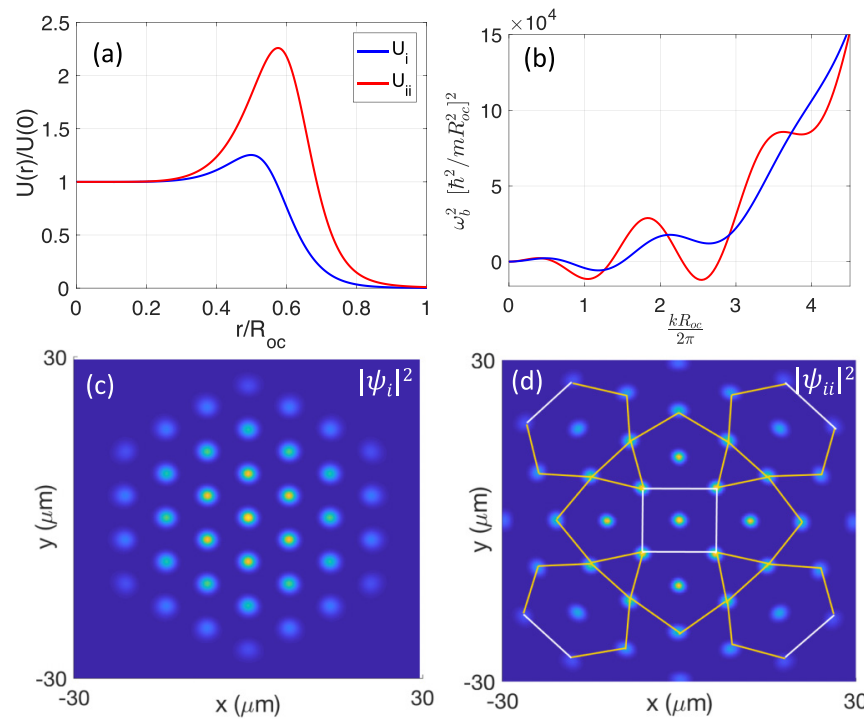

FIG. 5. Droplet quasicrystals. Adding a Gaussian to the conventional plateau in the $\mathrm{RnD}$ interaction profiles in (a) results in more than one roton unstable point in the Bogoliubov spectrum plotted in (b). Hence there will be more than one lattice constant that changes the conventional triangular lattice in (c) to a quasiordered crystal depicted in (d). (c), (d) The ground state of 2D GPE under potentials $U_{i}-U_{i i}$ are plotted. The dynamic parameters of $\left[U_{i}, U_{i i}\right]$ are $\Delta / 2 \pi=$ $-10 \mathrm{MHz}, \Omega_{2} / 2|\Delta|=1, \Omega_{1} / 2 \pi=[50,56] \mathrm{kHz}, n=100, \gamma_{1}=$ $\gamma_{2}=[35,70] \gamma_{p}$ with locked lasers, $U(r=0)=[900,750] \mathrm{Hz}$, and $N=200$ atoms. Further quasiordered patterns can be found in the SM.

multiple roton instabilities, leading to more than one lattice constant [see Fig. 5(b)]. This would develop quasiperiodic structured droplet crystals. The ground states associated with the potentials of Fig. 5(a) are plotted in Figs. 5(c) and 5(d) calculated by the GPE imaginary time evolution technique. More patterns can be found in the SM [61]. While the plots are depicted in 2D for the sake of presentation, the extension to $3 \mathrm{D}$ is trivial.

In conclusion, the laser linewidth used in the Rydberg dressing could be used as a controlling knob in tuning the interaction sign, strength, and shape. The possibility of changing the interaction sign by a laser linewidth opens different insights for interactions in AMO physics with an exclusive attractive or repulsive nature (e.g., Ref. [76]). Designing a hybrid potential profile, this Letter showed that $\mathrm{RnD}$ could be used for realizing 3D stable soliton molecules as well as quasiordered crystals. The level of control over the physical elements and exaggerated size and mass of these coherent molecules provide an ideal laboratory for the study of the molecules' chemistry in the transition from the microscopic to the macroscopic regime. The presented $\mathrm{RnD}$ potentials in Fig. 3 could also be used to test the quantum collision of heavy coherent systems at small speeds that is currently demanding to close the experimental hole in testing collapse models [77,78].

The author thanks T. Pohl and Y. Zhang for fruitful discussions and helpful comments. 
[1] D. Jaksch, J. I. Cirac, P. Zoller, S. L. Rolston, R. Cote, and M. D. Lukin, Fast Quantum Gates for Neutral Atoms, Phys. Rev. Lett. 85, 2208 (2000).

[2] M. D. Lukin, M. Fleischhauer, R. Cote, L. M. Duan, D. Jaksch, J. I. Cirac, and P. Zoller, Dipole Blockade and Quantum Information Processing in Mesoscopic Atomic Ensembles, Phys. Rev. Lett. 87, 037901 (2001).

[3] M. Saffman, T. G. Walker, and K. Mølmer, Quantum information with Rydberg atoms, Rev. Mod. Phys. 82, 2313 (2010).

[4] C. S. Adams, J. D. Pritchard, and J. P. Shaffer, Rydberg atom quantum technologies, J. Phys. B: At. Mol. Opt. Phys. 53, 012002 (2019).

[5] M. Khazali, C. Murry, and T. Pohl, Polariton Exchange Interactions in Multichannel Optical Networks, Phys. Rev. Lett. 123, 113605 (2019); M. Khazali, K. Heshami, and C. Simon, Photon-photon gate via the interaction between two collective Rydberg excitations, Phys. Rev. A 91, 030301(R) (2015).

[6] M. Khazali and K. Mølmer, Fast Multi-Qubit Gates by Adiabatic Evolution in Interacting Excited-State Manifolds of Rydberg Atoms and Superconducting Circuits, Phys. Rev. X 10, 021054 (2020).

[7] M. Khazali, Rydberg quantum simulator of topological insulators, arXiv:2101.11412.

[8] I. Friedler, D. Petrosyan, M. Fleischhauer, and G. Kurizki, Long-range interactions and entanglement of slow singlephoton pulses, Phys. Rev. A 72, 043803 (2005).

[9] A. V. Gorshkov, J. Otterbach, M. Fleischhauer, T. Pohl, and M. D. Lukin, Photon-Photon Interactions via Rydberg Blockade, Phys. Rev. Lett. 107, 133602 (2011).

[10] D. Tiarks, S. Schmidt-Eberle, T. Stolz, G. Rempe, and S. Dürr, A photon-photon quantum gate based on Rydberg interactions, Nat. Phys. 15, 124 (2019).

[11] D. Paredes-Barato and C. S. Adams, All-Optical Quantum Information Processing Using Rydberg Gates, Phys. Rev. Lett. 112, 040501 (2014).

[12] A. C. J. Wade, M. Mattioli, and K. Mølmer, Single-atom single-photon coupling facilitated by atomic-ensemble darkstate mechanisms, Phys. Rev. A 94, 053830 (2016).

[13] M. Khazali, K. Heshami, and C. Simon, Single-photon source based on Rydberg exciton blockade, J. Phys. B 50, 21 (2017); F. Ripka, H. Kübler, R. Löw, and T. Pfau, A room-temperature single-photon source based on strongly interacting Rydberg atoms, Science 362, 446 (2018).

[14] M. Khazali, Quantum information and computation with Rydberg atoms, Iranian Journal of Applied Physics 10, 19 (2021).

[15] N. Henkel, R. Nath, and T. Pohl, Three-Dimensional Roton Excitations and Supersolid Formation in Rydberg-Excited Bose-Einstein Condensates, Phys. Rev. Lett. 104, 195302 (2010).

[16] G. Pupillo, A. Micheli, M. Boninsegni, I. Lesanovsky, and P. Zoller, Strongly Correlated Gases of Rydberg-Dressed Atoms: Quantum and Classical Dynamics, Phys. Rev. Lett. 104, 223002 (2010).

[17] J. Honer, H. Weimer, T. Pfau, and H. P. Büchler, Collective Many-Body Interaction in Rydberg Dressed Atoms, Phys. Rev. Lett. 105, 160404 (2010).

[18] J. E. Johnson and S. L. Rolston, Interactions between Rydbergdressed atoms, Phys. Rev. A 82, 033412 (2010).
[19] C. Gaul, B. J. DeSalvo, J. A. Aman, F. B. Dunning, T. C. Killian, and T. Pohl, Resonant Rydberg Dressing of Alkaline-Earth Atoms via Electromagnetically Induced Transparency, Phys. Rev. Lett. 116, 243001 (2016).

[20] J. Zeiher, R. van Bijnen, P. Schauß, S. Hild, J.-Y. Choi, T. Pohl, I. Bloch, and C. Gross, Many-body interferometry of a Rydberg-dressed spin lattice, Nat. Phys. 12, 1095 (2016).

[21] I. Bouchoule and K. Mølmer, Spin squeezing of atoms by the dipole interaction in virtually excited Rydberg states, Phys. Rev. A 65, 041803(R) (2002).

[22] M. Khazali, H. W. Lau, A. Humeniuk, and C. Simon, Large energy superpositions via Rydberg dressing, Phys. Rev. A 94, 023408 (2016).

[23] M. Khazali, Progress towards macroscopic spin and mechanical superposition via Rydberg interaction, Phys. Rev. A 98, 043836 (2018).

[24] M. A. Norcia, A. W. Young, W. J. Eckner, E. Oelker, J. Ye, and A. M. Kaufman, Seconds-scale coherence on an optical clock transition in a tweezer array, Science 366, 93 (2019).

[25] L. I. R. Gil, R. Mukherjee, E. M. Bridge, M. P. A. Jones, and T. Pohl, Spin Squeezing in a Rydberg Lattice Clock, Phys. Rev. Lett. 112, 103601 (2014).

[26] T. Keating, C. H. Baldwin, Y.-Y. Jau, J. Lee, G. W. Biedermann, and I. H. Deutsch, Arbitrary Dicke-State Control of Symmetric Rydberg Ensembles, Phys. Rev. Lett. 117, 213601 (2016).

[27] F. Maucher, N. Henkel, M. Saffman, W. Królikowski, S. Skupin, and T. Pohl, Rydberg-Induced Solitons: ThreeDimensional Self-Trapping of Matter Waves, Phys. Rev. Lett. 106, 170401 (2011).

[28] F. Cinti, P. Jain, M. Boninsegni, A. Micheli, P. Zoller, and G. Pupillo, Supersolid Droplet Crystal in a Dipole-Blockaded Gas, Phys. Rev. Lett. 105, 135301 (2010).

[29] N. Henkel, F. Cinti, P. Jain, G. Pupillo, and T. Pohl, Supersolid Vortex Crystals in Rydberg-Dressed Bose-Einstein Condensates, Phys. Rev. Lett. 108, 265301 (2012).

[30] F. Cinti, T. Macri, W. Lechner, G. Pupillo, and T. Pohl, Defectinduced supersolidity with soft-core bosons, Nat. Commun. 5, 3235 (2014).

[31] A. Dauphin, M. Müller, and M. A. Martin-Delgado, Rydbergatom quantum simulation and Chern-number characterization of a topological Mott insulator, Phys. Rev. A 86, 053618 (2012).

[32] X. Li and S. Das Sarma, Exotic topological density waves in cold atomic Rydberg-dressed fermions, Nat. Commun. 6, 7137 (2015).

[33] A. W. Glaetzle, M. Dalmonte, R. Nath, I. Rousochatzakis, R. Moessner, and P. Zoller, Quantum Spin-Ice and Dimer Models with Rydberg Atoms, Phys. Rev. X 4, 041037 (2014).

[34] A. W. Glaetzle, M. Dalmonte, R. Nath, C. Gross, I. Bloch, and P. Zoller, Designing Frustrated Quantum Magnets with Laser-Dressed Rydberg Atoms, Phys. Rev. Lett. 114, 173002 (2015).

[35] R. M. W. van Bijnen and T. Pohl, Quantum Magnetism and Topological Ordering via Rydberg Dressing near Förster Resonances, Phys. Rev. Lett. 114, 243002 (2015).

[36] T. Keating, K. Goyal, Y.-Y. Jau, G. W. Biedermann, A. J. Landahl, and I. H. Deutsch, Adiabatic quantum computation with Rydberg-dressed atoms, Phys. Rev. A 87, 052314 (2013).

[37] T. Keating, R. L. Cook, A. M. Hankin, Y.-Y. Jau, G. W. Biedermann, and I. H. Deutsch, Robust quantum logic in neutral 
atoms via adiabatic Rydberg dressing, Phys. Rev. A 91, 012337 (2015).

[38] S. Möbius, M. Genkin, A. Eisfeld, S. Wüster, and J. M. Rost, Entangling distant atom clouds through Rydberg dressing, Phys. Rev. A 87, 051602(R) (2013).

[39] J. B. Balewski, A. T. Krupp, A. Gaj, S. Hofferberth, R. Löw and T. Pfau, Rydberg dressing: Understanding of collective manybody effects and implications for experiments, New J. Phys. 16, 063012 (2014).

[40] F. Nogrette, H. Labuhn, S. Ravets, D. Barredo, L. Béguin, A. Vernier, T. Lahaye, and A. Browaeys, Single-Atom Trapping in Holographic 2D Arrays of Microtraps with Arbitrary Geometries, Phys. Rev. X 4, 021034 (2014).

[41] T. Xia, M. Lichtman, K. Maller, A. W. Carr, M. J. Piotrowicz, L. Isenhower, and M. Saffman, RAndomized Benchmarking of Single-Qubit Gates in a 2D Array of Neutral-Atom Qubits, Phys. Rev. Lett. 114, 100503 (2015).

[42] A. Cooper, J. P. Covey, I. S. Madjarov, S. G. Porsev, M. S. Safronova, and M. Endres, Alkaline-Earth Atoms in Optical Tweezers, Phys. Rev. X 8, 041055 (2018).

[43] M. A. Norcia, A. W. Young, and A. M. Kaufman, Microscopic Control and Detection of Ultracold Strontium in OpticalTweezer Arrays, Phys. Rev. X 8, 041054 (2018).

[44] S. Hollerith, J. Zeiher, J. Rui, A. Rubio-Abadal, V. Walther, T. Pohl, D. M. Stamper-Kurn, I. Bloch, and C. Gross, Quantum gas microscopy of Rydberg macrodimers, Science 364, 664 (2019).

[45] S. Saskin, J. T. Wilson, B. Grinkemeyer, and J. D. Thompson, Narrow-Line Cooling and Imaging of Ytterbium Atoms in an Optical Tweezer Array, Phys. Rev. Lett. 122, 143002 (2019).

[46] Y. Wang, A. Kumar, T. Y. Wu, and D. S. Weiss, Single-qubit gates based on targeted phase shifts in a 3D neutral atom array, Science 352, 1562 (2016).

[47] D. Barredo, V. Lienhard, S. de Léséleuc, T. Lahaye, and A. Browaeys, Synthetic three-dimensional atomic structures assembled atom by atom, Nature (London) 561, 79 (2018).

[48] A. W. Glaetzle, R. M. W. van Bijnen, P. Zoller, and W. Lechner, A coherent quantum annealer with Rydberg atoms, Nat. Commun. 8, 15813 (2017).

[49] H. Weimer, M. Müller, I. Lesanovsky, P. Zoller, and H. P. Büchler, A Rydberg quantum simulator, Nat. Phys. 6, 382 (2010).

[50] M. Stratmann, T. Pagel, and F. Mitschke, Experimental Observation of Temporal Soliton Molecules, Phys. Rev. Lett. 95, 143902 (2005).

[51] P. Grelu, F. Belhache, F. Gutty, and J. M. Soto-Crespo, Phaselocked soliton pairs in a stretched-pulse fiber laser, Opt. Lett. 27, 966 (2002).

[52] K. Krupa, K. Nithyanandan, U. Andral, P. Tchofo-Dinda, and P. Grelu, Real-Time Observation of Internal Motion within Ultrafast Dissipative Optical Soliton Molecules, Phys. Rev. Lett. 118, 243901 (2017).

[53] P. Rohrmann, A. Hause, and F. Mitschke, Solitons beyond binary: Possibility of fibre-optic transmission of two bits per clock period, Sci. Rep. 2, 866 (2012).

[54] Z. Q. Wang, K. Nithyanandan, A. Coillet, P. Tchofo-Dinda, and $\mathrm{Ph}$. Grelu, Optical soliton molecular complexes in a passively mode-locked fibre laser, Nat. Commun. 10, 830 (2019).

[55] P. Grelu and N. Akhmediev, Dissipative solitons for modelocked lasers, Nat. Photonics 6, 84 (2012).
[56] B. Malomed, Bound solitons in the nonlinear Schrödinger/Ginzburg-Landau equation, Phys. Rev. A 44, 6954 (1991).

[57] N. Akhmediev, A. Ankiewicz, and J. M. Soto-Crespo, Multisoliton Solutions of the Complex Ginzburg-Landau Equation, Phys. Rev. Lett. 79, 4047 (1997).

[58] D. Y. Tang, W. S. Man, H. Y. Tam, and P. D. Drummond, Observation of bound states of solitons in a passively mode-locked fiber laser, Phys. Rev. A 64, 033814 (2001).

[59] G. Herink, F. Kurtz, B. Jalali, D. Solli, and C. Ropers, Realtime spectral interferometry probes the internal dynamics of femtosecond soliton molecules, Science 356, 50 (2017).

[60] A. V. Buryak, Y. S. Kivshar, M. F. Shih, and M. Segev, Induced Coherence and Stable Soliton Spiraling, Phys. Rev. Lett. 82, 81 (1999).

[61] See Supplemental Material at http://link.aps.org/supplemental/ 10.1103/PhysRevResearch.3.L032033 for further in-depth discussions about the dynamics of $\mathrm{RnD}$ interaction, interaction sign change, relaxation time, how the elements in the RnD spatial profile are formed, further decoherence sources including level mixing and anomalous broadening, illustrations of how the patterns of droplet quasicrystals could be controlled by manipulating the $\mathrm{RnD}$ interaction, and studies of the vulnerability of the phase-dependent soliton interaction to phase fluctuations.

[62] I. Seydi, S. H. Abedinpour, R. E. Zillich, R. Asgari, and B. Tanatar, Rotons and Bose condensation in Rydberg-dressed Bose gases, Phys. Rev. A 101, 013628 (2020).

[63] T. Macri, S. Saccani, and F. Cinti, Ground State and Excitation Properties of Soft-Core Bosons, J. Low Temp. Phys. 177, 59 (2014).

[64] O. N. Osychenko, G. E. Astrakharchik, Y. Lutsyshyn, Y. E. Lozovik, and J. Boronat, Phase diagram of Rydberg atoms with repulsive van der Waals interaction, Phys. Rev. A 84, 063621 (2011).

[65] D. Levine and P. J. Steinhardt, Quasicrystals: A New Class of Ordered Structures, Phys. Rev. Lett. 53, 2477 (1984).

[66] U. Grimm and M. Schreiber, Energy spectra and eigenstates of quasiperiodic tight-binding Hamiltonians, in Quasicrystals' Structure and Physical Properties, edited by H.-R. Trebin (Wiley-VCH, Weinheim, 2003).

[67] D. Tanese, E. Gurevich, F. Baboux, T. Jacqmin, A. Lemaitre, E. Galopin, I. Sagnes, A. Amo, J. Bloch, and E. Akkermans, Fractal Energy Spectrum of a Polariton Gas in a Fibonacci Quasiperiodic Potential, Phys. Rev. Lett. 112, 146404 (2014).

[68] M. Kohmoto and B. Sutherland, Electronic States on a Penrose Lattice, Phys. Rev. Lett. 56, 2740 (1986).

[69] T. Fujiwara and T. Yokokawa, Universal Pseudogap at Fermi Energy in Quasicrystals Phys. Rev. Lett. 66, 333 (1991).

[70] F. S. Pierce, S. J. Poon, and Q. Guo, Electron Localization in Metallic Quasicrystals, Science 261, 737 (1993).

[71] S. Wessel, A. Jagannathan, and S. Haas, Quantum Antiferromagnetism in Quasicrystals, Phys. Rev. Lett. 90, 177205 (2003).

[72] S. Kunze, R. Hohmann, H. J. Kluge, J. Lantzsch, L. Monz, J. Stenner, K. Stratmann, K. Wendt, and K. Zimmer, Lifetime measurements of highly excited Rydberg states of strontium I, Z. Phys. D 27, 111 (1993).

[73] M. Khazali, Applications of atomic ensembles for photonic quantum information processing and fundamental tests 
of quantum physics, Ph.D. thesis, University of Calgary, 2016.

[74] S. Sevincli, N. Henkel, C. Ates, and T. Pohl, Nonlocal Nonlinear Optics in Cold Rydberg Gases, Phys. Rev. Lett. 107, 153001 (2011).

[75] S. Stellmer, F. Schreck, and T. C. Killian, Degenerate quantum gases of strontium, in Annual Review of Cold Atoms and Molecules, edited by K. Madison, K. Bongs, L. D. Carr, A. M. Rey, and H. Zhai (World Scientific, Singapore, 2014), Vol. 2, pp. $1-80$.
[76] Y.-C. Zhang, V. Walther, and T. Pohl, Long-Range Interactions and Symmetry Breaking in Quantum Gases through Optical Feedback, Phys. Rev. Lett. 121, 073604 (2018).

[77] M. Toros and A. Bassi, Bounds on quantum collapse models from matter-wave interferometry: Calculational details, J. Phys. A 51, 11 (2018).

[78] M. Carlesso and A. Bassi, Current tests of collapse models: How far can we push the limits of quantum mechanics?, in Quantum Information and Measurement (QIM) V: Quantum Technologies, OSA Technical Digest (Optical Society of America, Washington, DC, 2019), paper S1C.3. 